\title{
Utilisation du foncier agricole à des fins de culture de Jatropha dans le Bassin arachidier sénégalais : une démarche controversée et des gains pas à la hauteur des attendus du programme
}

Utilização de propriedades agrícolas para fins de cultura de Jatropha no Bassin Arachidier senegalense: um processo controverso e ganhos que não atingem as expectativas do programa

Use of agricultural lands for Jatropha culture in the Bassin arachidier of Senegal:a controversial process and benefits below the program's expectations

Aminata Ndour

SaerSarr ${ }^{*}$

Fatoumata Signate $^{* * *}$

\section{Résumé}

Durant les années 2000, le secteur énergétique mondial a connu un tourment décisif du fait de la diminution des réserves, de la demande importante et surtout de la fluctuation des prix du pétrole. Le Sénégal, pays dépendant encore des énergies fossiles, s'est engagé dans une politique de diversification énergétique. Par conséquent, un programme biocarburant est initié. Le Bassin Arachidier est un des espaces sollicités pour l'application des politiques de promotion des biocarburants. Une zone du production agricole a accueilli une innovation énergétique. Ce fait marquant a guidé nos in-
* Est géographe. Elle effectue une thèse de doctorat à la Faculté des lettres et sciences humaines de l'Université Cheikh-Anta-Diop de Dakar sur la crise énergétique au Sénégal : enjeux et impacts de la culture du Jatropha Curcas (plante bioénergétique) sur le développement rural. Elle est accueillie comme doctorante au Bureau d'Analyses Macro-Economique (BAME) de l'Institut Sénégalais de Recherches Agricoles (ISRA) depuis 2009. E-mail: dia.aminta@gmail.com

** Est ingénieur agro-économiste. Il effectue un doctorat à l'université de Thiès, mention agronomie, sur la mécanisation agricole. Il est accueilli comme doctorant au Bureau d'Analyses Macro-Economiques (BAME) de l'Institut sénégalais de recherches agricoles (ISRA) depuis 2013. E-mail: saer_sarr@yahoo.fr

**** Doutora, Docente do Département de Géographie de l'Université Cheikh Anta Diop de Dakar (UCAD). E-mail: sfatima2000@live.fr

Recebido em 28/03/2017 - Aprovado em 10/05/2017 http://dx.doi.org/10.5335/hdtv.17n.2.7497 
terrogations sur les problèmes énergétiques qui ont incité la culture de Jatropha dans le Bassin Arachidier. De façon spécifique, notre analyse de la filière Jatropha précise les stratégies déployées. Il est questions dans ce cas d'étudier les effets de Jatropha sur l'espace de production et les conditions de vie des agriculteurs, des risques de réduction des superficies de cultures traditionnelles.

Mots clés: Biocarburant. Énergie. Effets. Jatropha. Spatiaux.

\section{Introduction}

En Afrique et partout ailleurs dans le monde, la terre constitue un patrimoine sacré fondamental pour les activités agricoles, elle constitue le facteur de production le plus important et par conséquent un levier incontournable pour la lutte contre la pauvreté. Ainsi, le foncier est au cœur des enjeux économiques, sociaux et politiques du développement de l'agriculture.

Aujourd'hui, la demande de terres pour la production agricole dans les pays africains a connu une accélération. ${ }^{1}$ Il existe, en même temps au niveau mondial, des pays riches qui ne disposent pas assez d'espace pour les cultures vivrières et qui dépendent du marché mondial pour s'approvisionner en produits alimentaires. Le renchérissement des prix des denrées alimentaires observée depuis les années 2000 a incité ces pays à rechercher des espaces de production afin d'assurer leur approvisionnement en produits alimentaires (MATHIEU, 2009). Aussi, le contexte de risque de pénurie des ressources énergétiques additionné à la volonté des dirigeants africains à intégrer la nouvelle dynamique de production des bioénergies a-t-il accru l'occupation des terres agricoles africaines par des investisseurs étrangers. Dans quelles conditions se déroulent ces conquêtes d'espaces?

Qui sont les principaux bénéficiaires? Quels seront les impacts spatiaux? Cette série de questions est intéressante à creuser pour appréhender la diversité de la question foncière. L'introduction des cultures bioénergétiques nécessite des investissements financiers, techniques et technologiques importants. Le Sénégal a pris ainsi l'option politique de solliciter des investisseurs étrangers afin de développer cette nouvelle filière des biocarburants. Ces derniers ont bénéficié de plusieurs centaines d'hectares de terres Or, l'une des contraintes majeures auxquelles l'espace agricole sénégalais est l'insuffisance des surfaces de culture.

Le rush vers la terre pour la production de bioénergies a conduit à des mouvements de délibération sur l'espace agricole du Sénégal. Ces acteurs ont beaucoup contribué à l'accentuation de la pression foncière partout dans le pays. Ainsi, d'importantes modifications sont opérées dans le système de production agricole, en particulier dans les espaces de production de plantes bioénergétiques; des cas de délibération de terres dans le bassin arachidier dans la Région de Kaolack et Thiès sont notés. Le bassin arachidier est un espace particulier au Sénégal du fait de ses potentialités agricoles. Traditionnellement, il est très sollicité pour 
la mise en œuvre d'innovations agricoles au Sénégal. Ce grenier du Sénégal a accueilli en 2008 une culture à vocation énergétique accompagnée d'acteurs étrangers. Une situation pourrait être un problème du fait de la cohabitation dans un même milieu de plusieurs types de spéculations aux fonctions différentes. Elle a ainsi suscité notre curiosité par rapport aux interactions qui pourraient découler de ce mixte proposé au secteur agricole sénégalais. Ce papier a pour objectif de montrer les attributions de terres à un nouveau système de production non-vivrière et leurs impacts sur les communautés locales dans le bassin arachidier

\section{Matériel et méthodes}

Ce travail a été réalisé dans les sites ciblés constituant les premières zones où Jatropha Curcas $L$ a été introduit. Les Régions de Kaolack, Kaffrine et Thiès, situées au cœur du bassin arachidier.

Carte 1 - Localisation des espaces étudiés

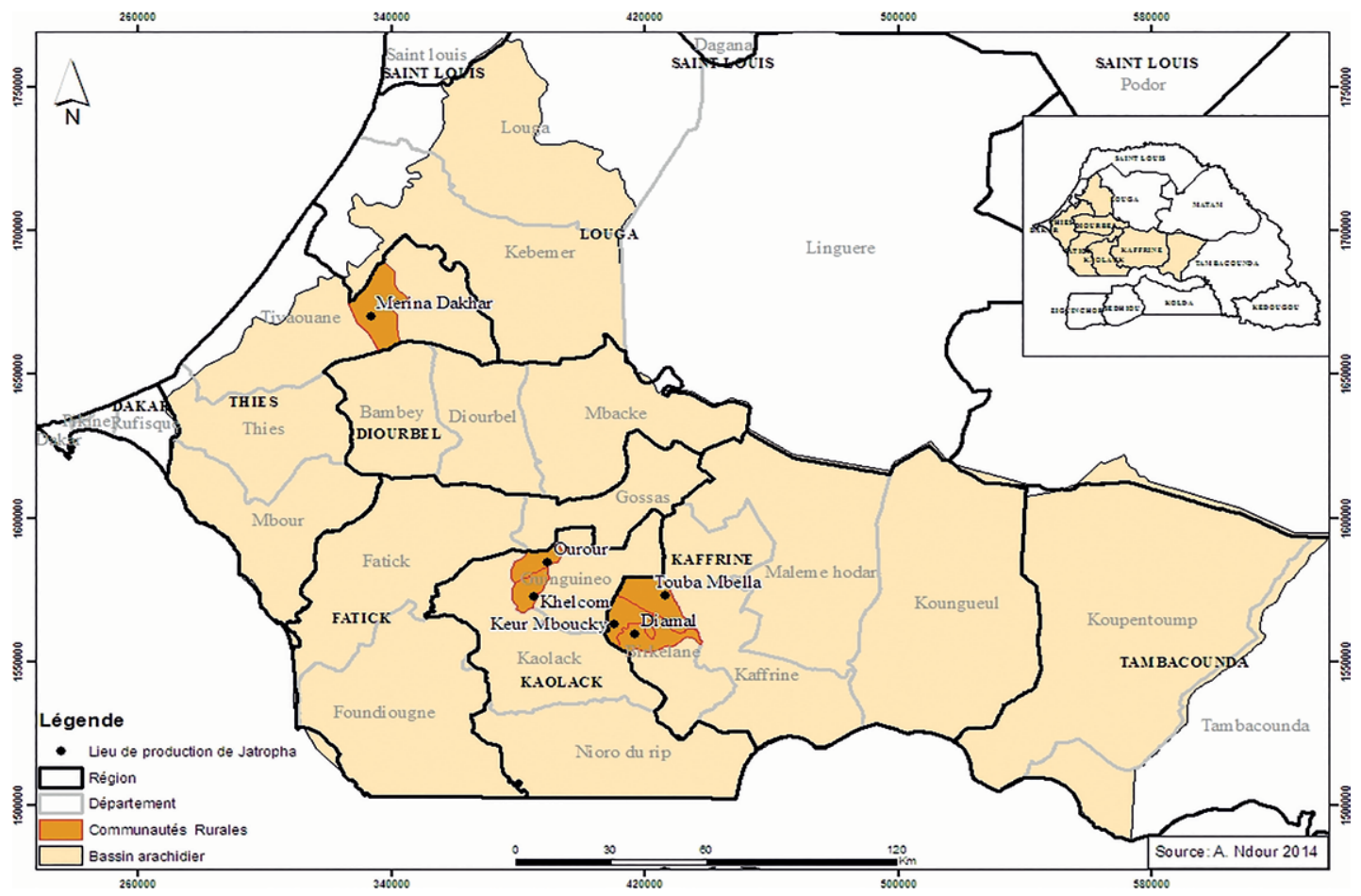

Source: (NDOUR ; CAMARA, 2014). 
Le choix des différents sites de production se justifie par une diversité des stratégies culturales relatives à la culture de Jatropha. Aussi, le paramètre de l'évolution de cette culture entre 2008/2009 a-t-elle profondément guidé nos choix des sites d'étude. Parce que Jatropha a été une nouvelle filière dans laquelle les premiers actes avaient été posés en 2002. Ainsi, nous avons ciblé la zone où les précurseurs ont été le plus concentrés. À Kaolack, Kaffrine et Thiès certaines Communes ${ }^{2}$ concernées par la culture de Jatropha Curcas L ont été ciblées.

La culture de Jatropha est assurée dans ces Communes choisies par des investisseurs privés qui, pour mener cette culture, ont formulé des demandes d'obtention de terres. $\mathrm{Au}$ niveau de ces zones de production, la cession de terres a eu lieu; en conséquence, les enjeux sur le foncier, sur le système agraire attirent notre attention et justifient notre choix.

Dans les trois Régions ciblées, nous avons obtenu un échantillon de 248 ménages répartis dans 20 villages. L'échantillonnage est effectué différemment selon les Régions:

À Kaolack, deux Communes sont ciblées dans lesquelles, les villages producteurs de Jatropha sont répertoriés. Le choix de l'échantillonnage est mené au tout début de l'introduction de Jatropha au Sénégal. Tous les villages qui en 2008/2009 avaient effectué une cession de terres et qui étaient déjà plantés sont concernés par ce papier. Au moment du choix des villages à étudier, il existait des villages qui avaient cédé des terres à des promoteurs privés, mais qui n'avaient pas été plantés et des villages qui n'avaient pas encore délibéré des terres pour Jatropha. En effet, dans chaque village ciblé, le $1 / 3$ du nombre de ménages total est tiré. L'enquête au niveau des ménages tirés a concerné aussi bien les ménages qui ont cédé des terres et ceux qui n'ont pas effectué de cession de terres pour Jatropha. Nous nous sommes focalisées sur l'indicateur de cession de terre car il n'existe pas de producteurs locaux à Kaolack ; la culture est assurée par des promoteurs privés. Les ménages sont concernés par la culture de Jatropha à travers la cession de terre et le recrutement des populations dans les exploitations de Jatropha.

A Kaffrine, un échantillonnage par un choix raisonné des villages est effectué avant l'administration du questionnaire. Un objectif de 100 ménages à enquêter était fixé ; ces ménages enquêtés sont proportionnels au nombre de ménages par village ciblé. Pour rappel à Kaffrine tous les villages enquêtés sont des villages dans lesquels il est noté l'existence d'un producteur local de Jatropha.

Une étude de cas a été également conduite pour disposer de données sur l'espace de culture de Jatropha Dans les Régions de Kaolack et Kaffrine. A Kaolack, deux Communes ont introduit Jatropha dans leur terroir; il s'agit de la Commune de Ourour et Khelcome. Les stratégies d'introduction de Jatropha dans ces Communes sont différentes ; l'espace consacré à Jatropha est plus important à Ourour qu'à Khelcome. Du coup, Ourour est choisi pour mener l'étude de cas pour une appréhension des effets de Jatropha sur l'espace et sur les populations locales. 
A Kaffrine, les trois Communes de la base mère sont choisies parce que le nombre de ménage impliqué dans la culture de Jatropha est très faible, comparé à Kaolack.

Pour mener des enquêtes sur les effets spatiaux de Jatropha, 30\% sont soustraits de la base existante ; ils sont répartis entre les Communes de Ourour à Kaolack, de Keur Mbouky, de Touba Mbella et de Diamal à Kaffrine. Un autre paramètre important pour justifier l'étude de cas est le fait que Jatropha est une filière inachevée. En conséquence, entre 2008 et 2014, l'évolution de la culture est très lente dans certaines communes. Les effets de la culture de Jatropha ne sont appréhendés que dans les espaces où l'on a remarqué une certaine évolution. La réalisation de l'étude de cas est subdivisée en trois parties: l'échantillonnage, l'administration du questionnaire sur le système agraire et les enquêtes par guide d'entretien.

Cet échantillonnage est fait sur la base d'un tirage effectué sur l'échantillon initial. En effet, pour une bonne représentativité, nous avons fait le choix raisonné de soustraire $30 \%$ des 248 chefs ménages qui constituent la base de données. Sur les 248 chefs de ménage, 53 sont concernés par l'étude de cas. Ces 53 chefs de ménages sont répartis entre les Communes de Ourour à Kaolack, de Keur Mbouky, de Touba Mbella et de Diamal à Kaffrine. Le nombre de ménage à enquêter dans chaque Commune est proportionnel au nombre de ménage interrogé durant les premières enquêtes ménage dans ces Communes ciblées, Pour chaque Commune, le tirage des ménages à interroger est aléatoire de même que les villages dans lesquels ils sont localisés. L'objectif de cet échantillonnage est de déterminer une population cible représentative de la population mère pour réaliser une cartographie des systèmes d'exploitation et étudier les effets sociospatiaux liés à la production de Jatropha.

A Ourour, sur les 75 ménages interrogés pour les enquêtes ménages, 22 sont tirés. Sur les 23 ménages, $42 \%$ soit 10 ménages sont constitués de ménages ayant cédé des terres à l'ANOC (African National Oil Corporation), la société privée étrangère productrice de Jatropha à Ourour. $58 \%$ de la base, soit 12 ménages sont tirés des ménages qui n'ont pas octroyé des terres aux investisseurs privés. À Kaffrine, il n'y a pas eu de cession de terre. En effet, $30 \%$ soit 30 ménages sont interrogés. Ainsi, nous avons obtenu pour le cas de Kaffrine 08 ménages sur les 26 interrogés à Keur Mbouky, 05 ménages sur les 17 ayant fait l'objet d'enquêtes à Diamal et en fin 18 ménages à Touba Mbella. À Kaffrine, un échantillon à choix raisonné est adopté. Ainsi, deux villages sont tirés au hasard dans chaque Commune rurale.

En effet, le choix des ménages et des villages à enquêter est effectué sur la base d'un échantillonnage aléatoire simple, tous les éléments de la population ont une égale chance d'être choisis, ils sont tirés au hasard. 


\section{Résultats}

a) Des terres agricoles attribuées à la culture de Jatropha, un rétrécissement des surfaces cultivées dans le bassin arachidier

La terre est très liée à la production ; sa gestion précède toutes les activités agricoles. Au Sénégal, les modèles d'acquisition de la terre jouent un rôle extrêmement important dans la cohésion des rapports sociaux. Le droit est attribué aux autochtones c'est-àdire aux populations originaires de la zone ; elles sont les premiers occupants de la terre par droit de la hache. Le capital foncier est détenu par les «Yale Lang» ou propriétaires fonciers ; ainsi, ils bénéficient du droit de s'approprier de la terre et de l'exploiter. En effet, ces familles anciennes, fondatrices de la zone, détiennent les terres, les transmettent selon les lignages. Dans le bassin arachidier, dans les communautés sérère, l'ethnie prédominante, la terre était transmise aux descendants par lignée matriarcale. Cependant, avec l'adoption des règles islamiques, les droits matrilinéaires laissent la place aux droits patrilinéaires ; la terre se transmet de père en fils (PELISSIER, 1966).

Dans les zones d'études, le principal mode de transmission de la terre est l'héritage, $80 \%$ des chefs de ménages ont acquis leur patrimoine foncier par héritage. Les chefs de ménages qui bénéficient de prêts et de dons sont moins importants du fait de l'insuffisance des superficies cultivables, ils sont estimés respectivement à $10 \%$ et $5 \%$.
Tableau 1-Mode d'attribution de la terre du bassin arachidier

\begin{tabular}{l|r|r}
\hline Mode d'accès à la terre & Effectifs & Pourcentages \\
\hline Délibération & 5 & $2 \%$ \\
Don & 13 & $5,2 \%$ \\
Héritage & 201 & $81 \%$ \\
Location & 3 & $1,2 \%$ \\
Prêt & 24 & $9,7 \%$ \\
Total & 248 & $100 \%$ \\
\hline
\end{tabular}

Source: (NDOUR ; DIA ; SAKHA, 2011).

Les changements induits par la culture de Jatropha sur l'espace et sur le système agraire seront analysés principalement dans la Commune de Ourour située dans la Région de Kaolack.

En outre, la production de Jatropha dans le bassin arachidier a conduit à des délibérations de terre. Ces attributions ont beaucoup contribué à l'accentuation de la pression foncière partout dans le pays. Ainsi, d'importantes modifications sont opérées dans le système de production agricole en particulier dans les espaces de production de plantes bioénergétiques comme Jatropha. "Cette situation découle du fait que les pays pauvres détenteurs de plus grandes réserves foncières sont de plus en plus sollicités par des investisseurs étrangers en quête de terres. Ces transactions foncières mondiales considérées par Chouquer comme un Commerce triangulaire» reflètent le déséquilibre existant entre les continents. Son Analyse démontre que les transactions foncières s'opèrent avec des vocations et intérêts spécifiques. «Des régions du monde ont, pour les unes, vocation à être exploitées pour nourrir; pour les autres, vocation à 
être nourries; pour les dernières, vocation à garantir» (CHOUQUER, 2011, p. 101).

De même, la terre est une ressource limitée, elle est également propriété privée et source de rente. Elle constitue une ressource primordiale pour la production agricole et est un bien socioéconomique important. La discrimination dans son accessibilité est de plus en plus notée entre producteurs privés détenteurs de moyens financiers et producteurs locaux représentés par les petites exploitations agricoles. Ainsi, le terme accaparement des terres est apparu suite aux délibérations importantes de terres à des promoteurs privés partout en Afrique et en Amérique Latine. Le FIDA, qualifie l'accaparement des terres comme un phénomène induit par des transactions foncières avec des promoteurs étrangers. Toutefois, «ces formes d'accaparement ne constituent qu'une petite part, les spoliations par les élites nationales restent considérables» (CCFD, 2012,). Au Sénégal, les autorités détiennent aussi une part non négligeable du patrimoine foncier. Ainsi, les années 2007/2008 sont particulièrement marquées par des politiques de relance des investissements dans le secteur agricole au Sénégal avec la mise en place de programmes. Ces différentes politiques de promotion du secteur agricole ont incité la possession des terres par les étrangers et par les élites. Toutefois l'utilisation abusive des forêts, l'avancée rapide de la sècheresse, la dégradation du couvert végétal sont les facteurs majeursquijustifientl'accaparement des terres.

En effet, une étude réalisée par le CCFTerre Solidaire sur les investissements et accaparement des terres et des ressources définit l'accaparement des terres par rapport aux conditions de cession, aux retombées sur les ressources et sur les populations.

l'accaparement des terres concerne la prise de contrôle d'un territoire (par achat ; location, occupation...), qu'elle soit légale ou non, qui entraînent des incidences négatives sur les communautés locales ou les usagers originaux du terrain, c'est à-dire lorsque les transactions foncières affectent directement ou indirectement le modèle économique, sociétal, social ou environnemental des communautés locales et portent donc atteinte aux droits inscrits dans la Charte internationale des droits de l'Homme (CCFD, 2012, p. 7).

Cette définition, renvoie à la situation notée au niveau du Bassin arachidier en particulier dans la commune de Ourour où des milliers d'hectares sont concédés à un promoteur privé. Ourour est une zone où l'agriculture et l'élevage constituent les principales activités exercées par les populations. La superficie cultivée en moyenne en 2006 est estimée à 10 hectares sur une superficie totale moyenne par chef de ménage estimée à 12 hectares. Une analyse de l'écart type calculé démontre les disparités notoires qui existent sur les proportions de terre détenues où l'écart type est supérieur à 7 . Ainsi, dans cette zone, il existe des chefs de ménages qui ont affecté des superficies importantes pour la production de Jatropha avec un maximum estimé à 28 hectares. Suivant les terres cédées, l'écart-type est estimé environ à 6 ; avec des minimums de cession enregistrés avoisinant 2 hectares; le maximum est égal 
à 19 hectares. Les superficies en moyenne cédées sont est estimées à 7 hectares.

Comme on pouvait $s^{\prime} y$ attendre, la Commune est confrontée à un recul progressif des surfaces disponibles pour les activités agricoles. Les superficies moyennes cultivées sont passées de 10,4 hectares avant l'introduction de Jatropha en 2005/2006 à 7,6 hectares en 2013. Les risques de compétition entre Jatropha et cultures vivrières sont de plus en plus pesants. Suivant l'utilisation de la terre, les risques sont en général associés à un accès plus faible des populations à la terre. La diminution des superficies de culture est récurrente dans les petites exploitations comprises entre 1 à 10 hectares. Plus les surfaces cultivées sont élevées, plus le niveau d'évolution des superficies entre 2006 et 2013 se rapproche. Ce rapprochement est continuel jusqu'à ce qu'il ait une parfaite égalité des superficies cultivées pour les grandes exploitations estimées environ à 21 hectares. Par ailleurs, la régression des terres cultivées résulte du fait que les cessions de terres effectuées par les petites exploitations sont importantes, ainsi, il y a eu des répercussions sur les surfaces cultivables et cultivées.

En effet, sur les $23 \%$ des chefs de ménage qui ont octroyé des terres aux producteurs privés de Jatropha pour toutes les zones étudiées, la Commune de Ourour occupe $15 \%$; il s'agit de la zone, où les cessions de terres ont été plus importantes ; les exploitations dont les superficies sont comprises entre 5 et 10 hectares représentent $70 \%$, ce qui témoigne de l'ampleur de l'acte de cession de terres chez les petits exploitants dans cette Commune.
Les ontmontréque, dansles Communes ciblées, 98\% des terres cédées par les chefs de ménage sont octroyées à des opérateurs privés étrangers. Exemple : 785 hectares sont cédés à Ourour dont 685 hectares mis en culture en 2009. Dans la Commune de Khelcome, 30 hectares sont cédés et mis en culture à Ndoubore et 35 à Saté Waly. Ces milliers d'hectares sont concédés à la culture de Jatropha. Ainsi, l'occupation de l'espace par une culture à vocation énergétique induit à des changements spatiaux importants en particulier à Ourour, la commune qui a consacré plus de terre à Jatropha. 
Carte 2 - Occupation des terres de culture par Jatropha à Ourour

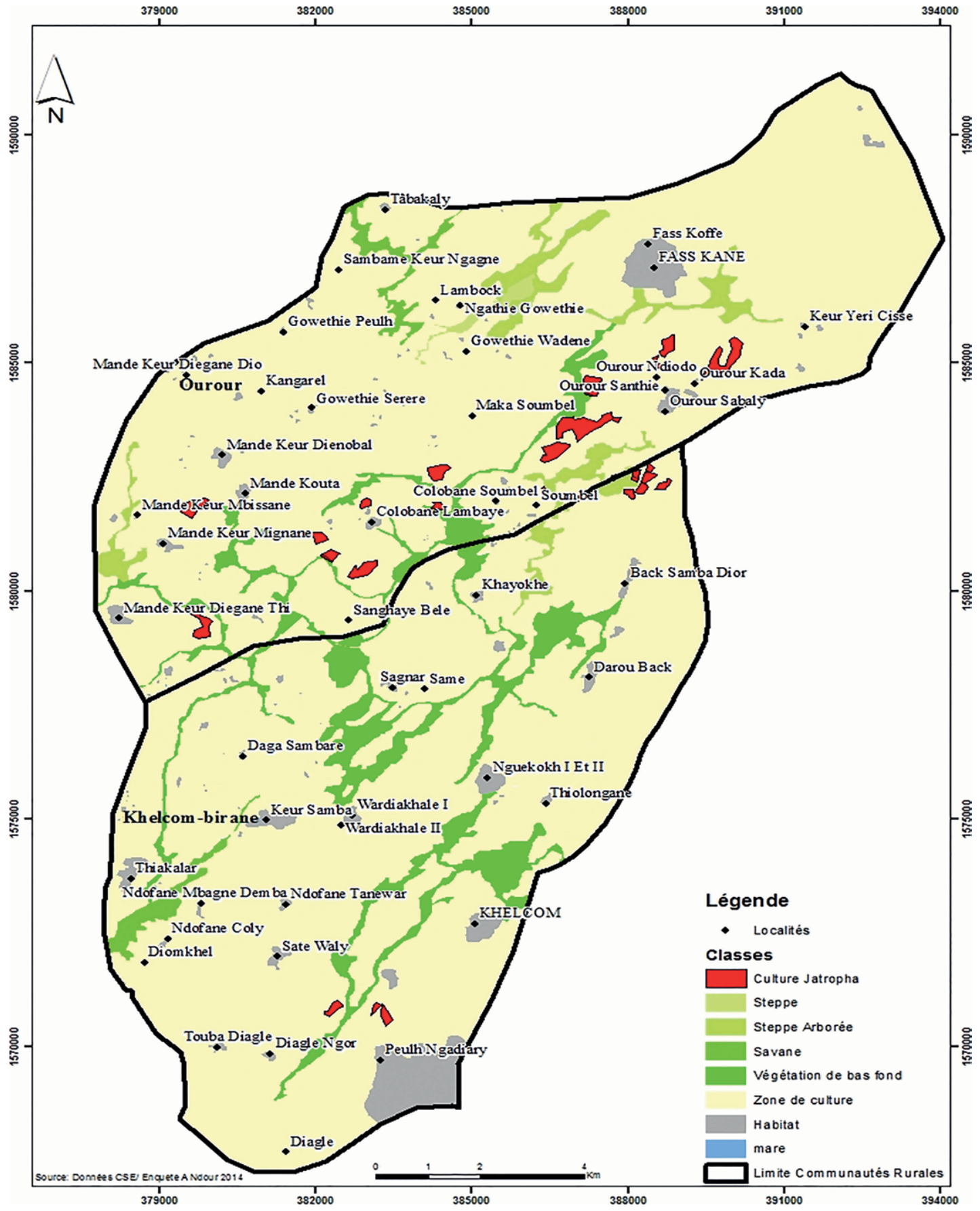

Source: (NDOUR ; CAMARA, 2014). 
De plus, l'introduction d'une nouvelle spéculation a contribué à reconfigurer l'espace agraire. L'utilisation du sol a connu un changement ; les terres qui sont aujourd'hui affectées à la culture de Jatropha étaient jadis occupées à 95\% en mil ; à 5\% en l'arachide. La situation observée à Ourour appuie la thèse de Lericollais qui consiste à dire : «Le paysage agraire se remodèle en fonction des pratiques agricoles nouvelles et objectifs de productions conçu dans le cadre d'une économie domestique de plus en plus des espaces et des ressources extérieurs» (LERICOLLAIS, 1987, p. 12).

Les surfaces constamment défrichées, nettoyées accentuent la disparition de la forêt. A Ourour, plusieurs terrains défrichés depuis 2009 ne sont toujours pas occupés du fait du taux de mortalité élevé de plantes de Jatropha. Ainsi, les centaines d'hectares défrichés à Ourour remodèlent l'espace, par une réaffectation d'espace pour les cultures traditionnelles terres. Les espaces consacrés aux cultures vivrières abritent maintenant Jatropha, désormais, son terroir agraire passe d'un système de culture diversifié à un système monocultural.

b) La cession des terres est à imputer à plusieurs facteurs

Le bassin arachidier a longtemps été la première région agricole du Sénégal. Il représentait $65 \%$ de la population rurale totale du pays en 1995 et $62 \%$ de la surface agricole utile (SAU) en 1998. Cependant, la région a subi de profondes mutations agraires avec le recul de la culture arachidière et les phases de sécheresses enregistrés dans les années 1968 à 1974 et au début des années
80. A partir de ce moment, les populations essaient de développer d'autres stratégies économiques dont les principales sont la migration et la diversification des activités agricoles. Donc, la culture Jatropha a émergé dans ce contexte précis malgré par une situation de précarité socio-économique.

La cession de terre pour la production de Jatropha implique uniquement les chefs de ménage âgés de $40-80$ ans ; $17 \%$ de ces chefs ont des âges compris entre 40 et 60 ans et $6 \%$ ont plus de 60 ans. Les raisons évoquées par ces personnes sont principalement liées à la pauvreté, à la recherche de revenus supplémentaires aux activités agricoles. La part importante des populations âgées peut être justifiée par le fait qu'en milieu rural, la terre est détenue par le chef de famille, qui exerce ses droits sur le patrimoine foncier familial. Ainsi, toute activité relative à la terre interpelle les responsables de la terre.

Les cessions sont effectuées parfois même, sans le consentement de tous les membres de la famille ; en conséquence, les frustrations s'accentuent chez les jeunes qui soutiennent être de tout temps opposés à l'idée d'un projet de production de Jatropha dans leur terroir. Ainsi, 100\% des chefs de ménages dont les âges compris entre 20-40 n’ont pas cédé des terres.

En effet, les cessions sont souvent source de litiges fonciers, surtout dans le domaine des partages des ressources en cas de décès du chef de ménage. En conséquence, la famille héritière est en conflit avec le projet de production de Jatropha. Le problème auquel ces populations sont confrontées est essentiellement lié au fait que les terres sont cédées sans contrat clair, écrit. Les 
réunions constituent les seules preuves des termes d'échanges qui ont lié les paysans au producteur étranger.

L'inexistence de protocole d'accord signé constitue une preuve des risques de perte de terres pour les chefs de ménage qui ont accepté de délibérer ou de vendre leur terre. Les populations sont pauvres, elles n'ont pas eu le niveau de formation requis pour pouvoir comprendre les stratégies des opérateurs. Le niveau d'étude est très faible pour tous les villages où les de terres cultivées en Jatropha excédent 45 hectares.

La taille des ménages n'influe pas directement sur la décision de céder des terres. Les cessions sont aussi importantes dans les ménages de petite taille comprise entre 5 et 10 personnes qu'au niveau des grands ménages qui comptent au moins 20 personnes. Des paramètres autres que le nombre de personnes qui composent le ménage déterminent les actes de cession. Ils dépendent de plusieurs facteurs dont la disponibilité des terres, la recherche de revenus supplémentaires, la pauvreté, la faible instruction des populations locales, l'absence de soutien de la part des autorités locales.

L'engouement qu'ont suscité les biocarburants, ainsi que les fausses idées véhiculées par les pouvoirs publics durant les premières expériences ont motivé certains paysans à la recherche de profit à prendre le risque de céder des terres. Les populations n'ont pas effectué des calculs, elles ont cédé par ce qu'elles croyaient en l'avenir des biocarburants afin d'améliorer leurs conditions de vie. En effet, tous les types de ménages sont impliqués, qu'il s'agisse de petits ménages sans ressources foncières importantes ou des ménages de grande taille qui disposent ou qui ne disposent pas assez de terres pour subvenir à leurs besoins alimentaires.

Les ménages monogames sont plus impliqués dans la cession de terres, sur les $23 \%$ des chefs de ménages qui ont cédé des terres, ils représentent $14 \%$. Le reste est constitué de ménages polygames avec $9 \%$. Les familles polygames disposent de plus de terres, plus de main d'œuvre et probablement plus de revenus. Les besoin de chercher des revenus supplémentaires pourraient être plus cruciaux chez les monogames de petite taille avec des disponibles non exploités du fait de déficit de main d'œuvre.

Le déficit de main d'œuvre locale causé essentiellement par l'exode massif, par la faiblesse des rendements agricoles, ont influencé les producteurs agricoles dans leur décision de mettre à la disposition des opérateurs privés des centaines d'hectares de terres pour la production de Jatropha. Les terres cédées sont selon ce graphique, en majorité, caractérisées par la faiblesse des superficies. Les exploitations familiales ne disposent pas suffisamment de terre de culture ; $60 \%$ des terres délibérées ont des superficies comprises entre 1 et 15 hectares. Les grandes cessions supérieures à 60 hectares sont, à cet effet, effectuées sur les terres où l'arachide était plus cultivée. Par exemple : la principale spéculation que Jatropha a remplacée est le mil. Ce dernier occupait essentiellement les terres délibérées dont les superficies sont estimées entre 1 et 59 hectares. Les délibérations de plus de 60 hectares sont occupées à 
5\% par le mil. Cependant, les cessions de terres où jadis on pratiquait l'arachide ont concerné les terres dont les superficies sont supérieures à 60 hectares. Ces types de cession sont enregistrés uniquement dans des villages localisés dans la Commune de Ourour, où les cessions de terres dépassent 700 hectares.

Carte 3 - Délibération des terres à Ourour en 2008

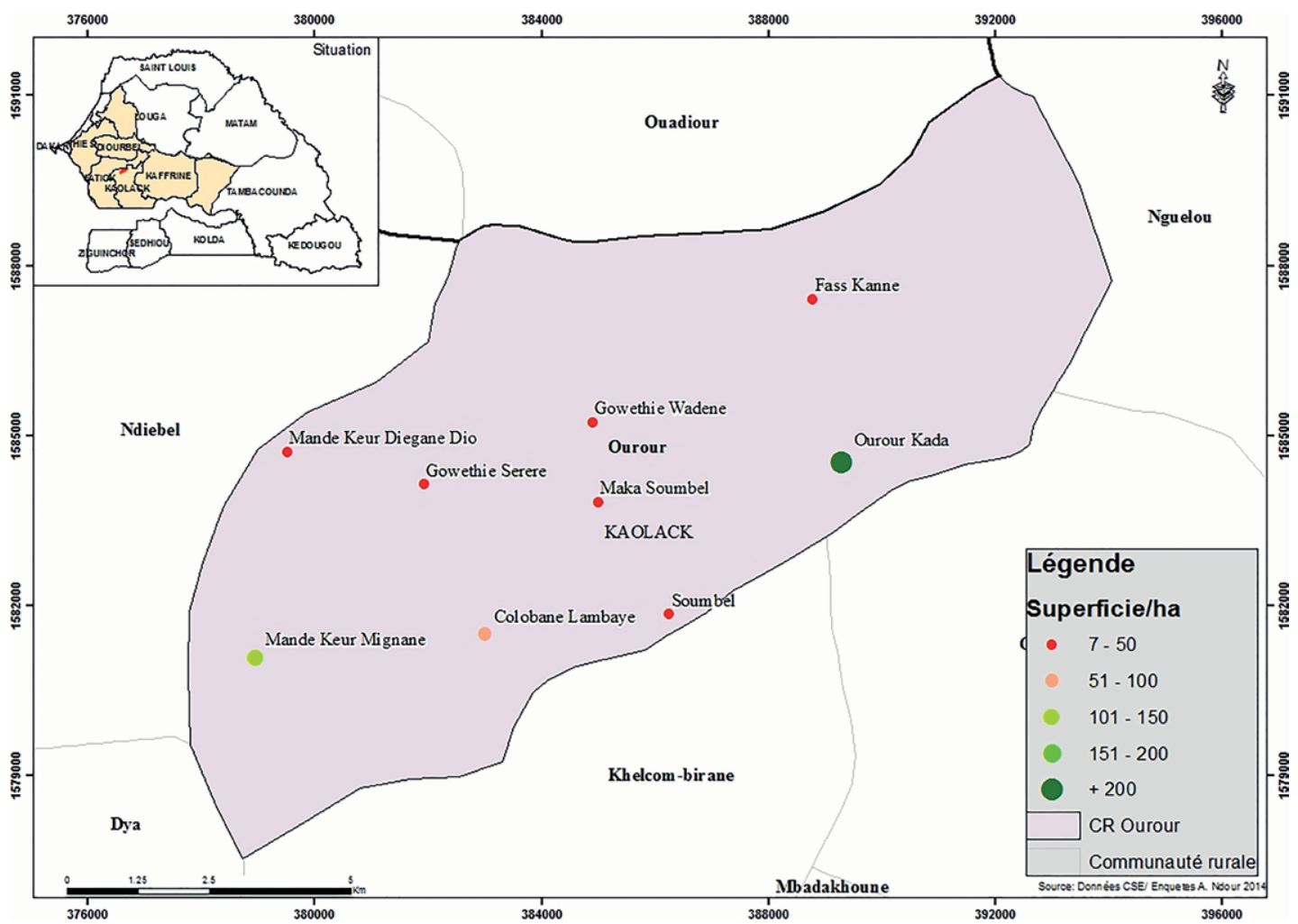

Source: (NDOUR ; CAMARA, 2014).

La carte ci- dessus, montre la part importante de terres cédées à Ourour pour la production de Jatropha. Parmi les chefs de ménage qui n'ont pas octroyé de terres, ceux qui ont évoqué le manque de terre représentent la majorité avec $35 \%$. Ce chiffre démontre une adhésion importante des populations pour l'introduction de Jatropha dans leur terroir au début du projet d'implantation. Ainsi, plusieurs chefs des petites exploitations ont déclaré avoir cédé une partie de leur patrimoine foncier pour disposer des opportunités de travail.

Par ailleurs, une analyse multivariée entre cession de terre et situation matrimoniale montre l'absence totale de la catégorie des divorcés et des veufs dans la cession de terre. Ils ne sont pas détenteurs de terre. Ils peuvent disposer de terres mais en général les superficies sont tellement faibles 
qu'ils écartent toute possibilité des legs ou de cession de terre. En effet, 100\% des veufs rencontrés sur le terrain ont déclaré n'avoir pas effectué des cessions de terre; les délibérations ont surtout concerné les chefs de ménages monogames et polygame cf tableau ci-dessus. Les populations qui ont acquis des terres par délibération des autorités administratives ou par héritage ont le pouvoir de décision sur les ressources foncières.

Par ailleurs, un autre facteur influent sur les actes de cession de terre est le niveau d'étude des chefs de ménages. Nos enquêtes ont montré que la proportion de chefs de ménage qui n'ont pas de niveau d'étude élevé est importante parmi ceux qui ont cédé des terres. Ils sont estimés à $45 \%$. Les cessions de terre comprises entre 1 et 15 hectares sont effectuées dans des villages où $40 \%$ des chefs de ménages ont fréquenté l'école coranique, $5 \%$ ont suivi une formation en alphabétisation.

Cependant, la proportion de chefs de ménage qui n'ont pas fréquenté l'école est estimée à $10 \%$ dans les villages où les superficies cultivées en moyenne sont comprises entre 1 et 5 hectares. Aussi, les surfaces qui excédent 50 hectares et qui sont consacrées au Jatropha sont-elles localisées dans les villages où le niveau d'étude est quasiment nul.

En outre, le disponible en terre de culture influe sur les actes de cession parce que toutes les terres cédées à Ourour appartiennent à des exploitants dont les superficies cultivées sont comprises en moyenne entre 5 et 10 hectares. La cession des terres est ainsi un acte effectué essentiellement par les petits producteurs agricoles.

A Ourour, 15\% des exploitations familiales de l'échantillon ont effectué des cessions de terre, $85 \%$ n'ont pas cédé des terres à L'ANOC. Les exploitations qui cultivent en moyenne 5 à 10 hectares sont majoritaires à Ourour, elles représentent $70 \%$. Celles dont les superficies moyennes mises en valeur sont comprises entre 10 et 20 hectares restent faibles, elles représentent $30 \%$. Les exploitations qui cultivent des superficies assez élevées pouvant atteindre 20 hectares ne sont pas impliquées dans les attributions de terre. La nécessité de vendre des terres pour disposer de revenus supplémentaires constitue le motif principal des legs opérés sur l'espace agricole à Ourour. La pauvreté est à cet effet au cœur des transactions entre opérateurs privés et populations locales. Durant nos recherches, Un enquêté (VD) pour justifier et démontrer qu'ils ont été abusés, disait : «niak bagnoul dara, niak rek mo waral li ame téye» ${ }^{3}$ pour dire qu'un pauvre ne peut rien refuser, la pauvreté constitue la principale cause des litiges fonciers vécus.

\section{Quel impact? La contrepartie financière est marginale}

Les indemnités perçues suite à la cession de terre par les populations soulèvent des interrogations, quant aux avantages que les populations peuvent en tirer. Les cessions sont à $90 \%$ effectuées par substitution aux espaces de cultures vivrières qui ont toujours utilisé les terrains de sol sableux ou sol ferrugineux tropicaux. 
Toutes les Communes ciblées présentent la même structure pédologique. Il s'agit de «sols dior « affectés traditionnellement aux cultures de mil et arachide. Les terres consacrées à Jatropha sont retirées des terrains de cultures des petites et moyennes exploitations. L'introduction de cette plante est une opportunité de diversification des activités et de création d'emploi, mais, les revenus tirés de ces terres ne sont pas permanents. 95\% sont des employés sont des temporaires; la Rémunération inférieure à 2000 FCFA par jour (3 euros).

L'obtention d'emploi étant la condition convenue avec l'opérateur pour la cession des terres, cet emploi remet en cause les effets positifs de la culture sur les conditions de vie des populations.

Même pour ceux qui sont recrutés, $80 \%$ des ouvriers ont déclaré les temps qu'ils ont travaillé dans le projet est inférieur à 2 années ; rares sont les employés recrutés en permanence. A Ourour, nos enquêtes ont montré que parmi tous ceux qui ont cédé des terres, deux seulement ont un contrat clair permanent avec le projet. En effet, l'instabilité de l'emploi qui se répercute sur les revenus des populations qui ont délibéré des terres est à l'origine de l'accroissement de la précarité dans cette commune.

Les chefs de ménage qui se sont retrouvés dans cette situation sont nombreux, ils sont estimés à plus de $60 \%$. Ils ont tous effectué des cessions de terres importantes mais, le projet de production de Jatropha ne leur procure pas assez de revenus pour améliorer leurs conditions de vie. C'est le cas de M. N., 61 ans, un paysan déçu du projet de production de Jatropha à Ourour.
Un chef d'exploitation agricole, né à Ourour, a été sollicité pour les cessions de terre. Une des conditions du contrat avec l'opérateur était le recrutement pour 6 hectares cédés. Rassuré des négociations, il cède 20 hectares de terres au projet qui représentent quasiment la totalité de son patrimoine foncier. Le salaire proposé selon ses proposé ; à l'assemblée générale tenue est fixé à 75 000. Le protocole d'accord privilégiait les populations qui ont cédé des terres. Enfin, les recrutements sont effectués sans prendre compte le nombre d'hectare cédé. Ainsi, il se retrouve avec un seul employé dans le projet de production de Jatropha à Ourour avec une indemnisation de 45000 francs le mois. Un montant très contesté par la suite. Aujourd'hui, ce vieux vit une situation difficile $d u$ fait qu'il a perdu ses terres et que les ressources tirées de Jatropha sont insuffisantes et instables. ${ }^{4}$

Pour toutes ces raisons, la cession de terre de culture pour Jatropha remet en cause la rentabilité de Jatropha en termes de revenu dans le bassin arachidier. Ainsi, la cohésion sociale connait des changements, la population se subdivisent ainsi en plusieurs groupes. Mais en réalité; ce qui ne change pas dans les stratégies de gestion c'est que la terre est un patrimoine communautaire partout dans les pays ; sa gestion est communautaire et sacrée. Le chef de terre ou «yaal lang» en milieu sérère sert d'intermédiaire entre les membres de la communauté et la terre. Il joue ainsi un rôle important; car, il exerce son autorité sur toutes les activités liées à la terre. Toutefois, l'introduction de Jatropha, une culture de rente à vocation non alimentaire a perturbé d'avantage les règles de gestion de la terre. Également, sa promotion a occasionné une perte importante du patrimoine foncier au niveau du bassin arachidier. 


\section{Conclusion}

La Culture Jatropha est très conjointe au foncier. Aujourd'hui, elle requière une disponibilité de la terre, qui constitue le premier facteur de production. Les enjeux fonciers liés à la culture de Jatropha sont au cœur des préoccupations des investisseurs du secteur de production des biocarburants. En effet, les demandes en terre sont tellement importantes qu'il y a eu des répercussions sur l'évolution des espaces de culture depuis l'introduction de Jatropha dans les zones ciblées par cette étude (Kaolack, Kaffrine). Ces répercussions n'existent que dans les espaces où Jatropha est cultivé en plein champ ou en association aux cultures vivrières. Ce mode de production a incité la cession de terre qui, à notre avis, est le principal problème de la culture de Jatropha. Ce sont des terres de culture qui sont consacrées au Jatropha, particulièrement à Ourour où des centaines d'hectares sont délibérés à un opérateur privé étranger. Les enjeux fonciers sont, à cet effet, déterminants dans les relations entre éleveurs, producteurs de Jatropha et agriculteurs.

Une analyse de la situation de la production de Jatropha à Kaffrine en corrélation avec le disponible terrien montre que Kaffrine est une Région qui n'a pas subi une perturbation de ses espaces de cultures. L'introduction de ces cultures bioénergétiques n'a pas eu d'effets visibles. Mais, les producteurs locaux ont des difficultés à maintenir la plante en vie, ainsi, beaucoup d'entre eux ont laissé disparaître les haies vives; la moitié des haies plantées ont connu un échec.
Resumo

Nos anos 2000, o setor energético mundial vivenciou uma situação crítica em razão da redução de reservas, do aumento da demanda e, sobretudo, da flutuação dos preços do petróleo. O Senegal, país dependente ainda de energias fósseis, engajou-se numa política de diversificação energética. Desse modo, um programa biocarburante teve início. $\mathrm{O}$ Bassin Arachidier é um dos espaços escolhidos para a aplicação de políticas de promoção de biocarburantes. Uma zona de produção agrícola incorporou essa estratégia de inovação tecnológica. Essa questão marcante direcionou nossas interrogações sobre os problemas energéticos que têm promovido a cultura de Jatropha no Bassin Arachidier. Especificiamente, nossa análise do estudo da cadeia produtiva da Jatropha evidencia as estratégias desenvolvidas, dentre elas, os seus efeitos no espaço de produção, as condições de vida dos agricultores e os riscos da redução de culturas tradicionais.

Palavras-chave: Biocarburante. Consequências espaciais. Energia. Jatropha.

\section{Abstract}

In the 2000s, the world's energy sector went through a critical situation due to decreasing reserves, increasing demand and especially to the oscillation of oil prices. Senegal, a country still dependent on fossil fuels, engaged in a policy of energy diversification. Thus, a biofuel program was begun. The Bassin Arachi- 
dier is one of the areas chosen to apply the biofuel promotion policy. A zone of agricultural production incorporated this strategy of technological innovation. This important matter directed our questions on the energetic problems that have promoted the Jatropha culture in the Bassin Arachidier. Specifically, our analysis of the study of the Jatropha production chain shows the strategies developed, among them their effect on the production space, farmers' life condition and the risks of decrease in traditional cultures.

Keywords: Biofuel. Energy. Jatropha. Spatial consequences.

\section{Notes}

1 La demande de terre n'est pas un phénomène totalement nouveau. Il y avait déjà, par exemple, de grandes compagnies agro-exportatrices comme Dole et Chiquita en Amérique Centrale, ou Unilever - le palmier à huile - en Afrique Centrale. Mais ce qui est nouveau c'est qu'il y a une accélération et d'autres acteurs qui arrivent. "Pour Paul Mathieu, de réelles opportunités existent, mais uniquement à condition que les accords de cession des terres soient bien négociés et effectués dans un souci de transparence, tant de la part des pays investisseurs que de la part des pays receveurs. Car opportunité rime avec responsabilité" (MATHIEU, 2009).

2 L'appellation "Commune" est utilisée dans le document en lieu et place de "Communauté rurale" compte tenu des mutations intervenues à partir de 2014; les communautés rurales sont passées communes.

3 Témoignage aux auteurs.

4 Témoignage aux auteurs.

\section{Bibliographie}

CCFD. Investissements et accaparements des terres et des ressources : prévenir les violations des droits humains. 2012. Disponible en : <ccfd-terresolidaire.org/IMG/pdf/acc_terres_ccfdjuin2012.pdf>. Accès dans : 20 févr. 2017.

CHOUQUER, G. Le nouveau commerce triangulaire mondial ou les analogies $\mathrm{du}$ foncier contemporain. Études Rurales, Paris, v. 187, n. 37, p. 95-130, 2011.

LERICOLLAIS, A. Analyse du changement dans les systèmes agraires sérères au Sénégal : bilan et perspectives de recherches. Marseille: Orstom, 1987.

MATHIEU, P. Comment Réagir Aux acquisitions de terres? Renforcer les capacités de négociation des États. Défis Sud., Bruxelas, n. 89, p. 14-15, juin/juil. 2009.

NDOUR, A.; DIA (D.) Fall, C. S.; SAKHA, J. M. S. Le Sénégal face à la crise énergétique mondiale: Enjeux de l'émergence de la filière des biocarburants. Sud Sciences \& Technologies, Dakar, n. 19 \& 20, p. 69-80, 2011.

NDOUR, A. D.; CAMARA, A. D. Accompagnement des femmes dans l'adaptation au changement climatique. 2014. Disponible en : <https:/ / portal.mtt.fi/portal/page/portal/ mtt_en/projects/foodafrica/news/Accompagnement $\% 20$ des $\% 20$ femmes $\% 20$ dans $\% 20$ $1 \%$ E2\% $80 \%$ 99adaptation $\% 20$ au \% 20changement $\% 20$ climatique $>$. Accès dans : 21 févr. 2017.

PELISSIER, P. Les paysans du Sénégal : les civilisations agraires du Cayor à la Casamance. Saint-Yriex, Fabregne: CNRS, 1966. 